Revista Brasileira de Agricultura Irrigada v.14, nº.3, p. 4058 - 4070, 2020

ISSN 1982-7679 (On-line)

Fortaleza, CE, INOVAGRI - http://www.inovagri.org.br

DOI: $10.7127 /$ rbai.v14n101163

Protocolo 1163.20 - 05/05/2020 Aprovado em 29/10/2020

\title{
COMPARAÇÃO ENTRE MÉTODOS DE ESTIMATIVA DA EVAPOTRANSPIRAÇÃO DE REFERÊNCIA NO OESTE BAIANO E MESORREGIÃO PAULISTA
}

\author{
Rodrigo Junior Nandorf ${ }^{1}$, Rudson Tonoli Feisberto ${ }^{1}$, André Dalla Bernardina Garcia ${ }^{2}$, Gustavo \\ Haddad Souza Vieira ${ }^{3}$, Paola Alfonsa Lo Monaco Vieira ${ }^{4}$, Alberto Chambela Neto ${ }^{5}$
}

\begin{abstract}
RESUMO
Considerando a importância econômica das culturais anuais irrigadas da Mesorregião de Piracicaba e do Oeste Baiano, os métodos de estimativas da evapotranspiração tornam-se imprescindíveis para o manejo eficiente do fornecimento de água via irrigação. O objetivo do presente estudo foi avaliar o desempenho dos métodos Hargreaves-Samani, Radiação Solar FAO, Makkink e Jensen-Haise em comparação ao método padrão Penman-Monteith FAO56 para os municípios de Piracicaba - SP e Luís Eduardo Magalhães - BA, em escala anual e sazonal. A comparação se baseou nos parâmetros da regressão linear $\left(\beta_{1}\right.$ e $\left.\beta_{0}\right)$, coeficiente de determinação, índice de concordância de Willmott, coeficiente de correlação, coeficiente de confiança e raiz quadrada média do erro. $\mathrm{O}$ município baiano apresentou maior evapotranspiração diária em relação ao paulista com valores de 5,4 e 3,4 $\mathrm{mm} \mathrm{dia}{ }^{-1}$, respectivamente. Os métodos mais indicados para estimativa da evapotranspiração de referência para Piracicaba-SP e Luís Eduardo Magalhães-BA são Makkink e Radiação Solar FAO, respectivamente, tanto em escala anual quanto sazonal. Hargreaves-Samani torna-se o menos recomendado para estimativa da Evapotranspiração de Referência (ET ${ }_{0}$ em Luís Eduardo Magalhães. Já em Piracicaba, o método Hargreaves-Samani pode ser utilizado, principalmente pela sua simplicidade.
\end{abstract}

Palavras-chave: necessidade hídrica, Penman-Monteith, irrigação, estações do ano.

\section{COMPARISON BETWEEN METHODS TO ESTIMATION OF REFERENCE EVAPOTRANSPIRATION IN THE WEST BAHIA AND PAULISTA MESORREGION}

\footnotetext{
${ }^{1}$ Graduando em Agronomia, Instituto Federal do Espírito Santo/campus Santa Teresa, Santa Teresa, Espírito Santo. Email: rodrigojrnandorf@gmail.com, rudsontonoli@gmail.com

${ }^{2}$ Mestrando em Produção Vegetal, Universidade Estadual do Norte Fluminense, Campos dos Goytacazes, Rio de Janeiro. E-mail: andredallabg@outlook.com

${ }^{3}$ Dr. em Engenharia Agrícola, Instituto Federal do Espírito Santo/campus Santa Teresa, Santa Teresa, Espírito Santo. Email: ghsvieira@gmail.com

${ }^{4}$ Dra. em Engenharia Agrícola, Instituto Federal do Espírito Santo/campus Santa Teresa, Santa Teresa, Espírito Santo. Email: paolalomonaco2004@yahoo.com.br

${ }^{5}$ Dr. em Ciência Animal, Instituto Federal do Espírito Santo/campus Santa Teresa, Santa Teresa, Espírito Santo. E-mail: chambela@gmail.com
} 


\begin{abstract}
Considering the economic importance of the annual irrigated crops in the Piracicaba region and the West of Bahia state, the methods of estimating evapotranspiration become essential for the efficient management of water supply by irrigation. The objective of the present study was to evaluate the performance of the Hargreaves-Samani, Solar Radiation FAO, Makkink and Jensen-Haise methods compared to the standard Penman-Monteith FAO56 method for the municipalities of Piracicaba - SP and Luís Eduardo Magalhães - BA, Brazil, on an annual and seasonal scales. The comparison was based on the linear regression parameters $\left(\beta_{1}\right.$ e $\left.\beta_{0}\right)$, determination coefficient, Willmott agreement index, correlation coefficient, confidence coefficient, and the root mean square error. The municipality of Bahia presented higher daily evapotranspiration compared to São Paulo, with values of 5.4 and $3.4 \mathrm{~mm} \mathrm{day}{ }^{-1}$, respectively. The most suitable methods for estimating reference evapotranspiration for Piracicaba - SP and Luís Eduardo Magalhães - BA are Makkink and Solar Radiation FAO, respectively, both on an annual and seasonal scales. Hargreaves-Samani becomes the least recommended for estimating Reference Evapotranspiration (ETo) in Luís Eduardo Magalhães. In Piracicaba, the H-S method can be used, mainly for its simplicity.
\end{abstract}

Keywords: water needs, Penman-Monteith, irrigation, seasons.

\section{INTRODUÇÃO}

Atualmente, o uso racional de recursos hídricos está pautado em vários encontros mundiais e estudos científicos. A demanda de água, principalmente para a irrigação, tornouse imprescindível na agricultura. No nível global, as taxas de retirada são de 69\% agrícola (incluindo irrigação, pecuária e aquicultura), $12 \%$ municipal (incluindo doméstico) e 19\% industrial (FAO, 2016).

A irrigação torna-se necessária para suprir as necessidades hídricas de acordo com os estádios fenológicos das culturas. Segundo Paulino et al. (2019), as mudanças climáticas nas variáveis meteorológicas afetam a evapotranspiração e, consequentemente, promovem a necessidade de alternativas de manejo eficiente dos recursos hídricos. Em áreas agrícolas que apresentam climas áridos e semiáridos ou durante estações secas prolongadas, a irrigação é necessária para compensar o déficit de evapotranspiração (ET) devido à precipitação insuficiente.

A agricultura brasileira apresenta regiões que são referências na produção de culturas anuais, onde o manejo da irrigação deve proporcionar uso sustentável dos recursos hídricos. A região Oeste da Bahia possui 116 mil hectares irrigados, com predominância de pivôs centrais no cultivo de algodão, apresentando potencial hídrico para o aumento de área irrigada, principalmente para o município de Luís Eduardo Magalhães, comprovado pela existência de rios perenes e do aquífero Urucuia (A.I.B.A., 2013).

Além da região oeste baiana, a mesorregião de Piracicaba é destaque pelo cultivo de culturas anuais, especialmente de cana-de-açúcar, onde lavouras paulistas apresentam déficit hídrico durante $o$ ano conforme verificado por Monteiro (2012), necessitando assim de irrigação e manejo eficiente.

Para conhecer a evapotranspiração das culturas anuais, principalmente para quantificar a água a ser aplicada através da irrigação, é necessário conhecer previamente a evapotranspiração de referência $\left(\mathrm{ET}_{0}\right)$. Neste contexto, a $\mathrm{ET}_{0}$ é um parâmetro básico e de extrema importância para proporcionar a otimização do uso de recursos hídricos, tornando-se um fator essencial no que tange o uso eficiente de água para a agricultura (ANTONOPOULOS; ANTONOPOULOS, 2017).

Desta forma, sugere-se a realização de análises relacionadas ao manejo hídrico, como a estimativa da evapotranspiração de referência $\left(\mathrm{ET}_{0}\right)$, com o intuito de fornecer ferramentas para apoio na tomada de decisão e otimizar o uso da água na agricultura por meio 
de uma gestão mais sustentável, fundamentada na coleta e cálculo de séries históricas de dados climáticos diários.

Diante do exposto, o presente trabalho objetivou avaliar o desempenho de diferentes métodos de estimativa da evapotranspiração de referência utilizando dados meteorológicos baseados em medições diárias ao longo de um período de cinco anos, com análise sazonal e anual de quatro métodos diferentes comparados com o método padrão Penman Monteith - FAO 56, nos municípios de Piracicaba, na região central de São Paulo (SP) e Luís Eduardo Magalhães, no oeste da Bahia (BA).

\section{MATERIAL E MÉTODOS}

A análise foi realizada com dados meteorológicos do Posto Meteorológico da Escola Superior de Agricultura Luiz de Queiroz (ESALQ) e Posto Meteorológico de Luís Eduardo Magalhães. Para ambas localidades as variáveis meteorológicas utilizadas foram: temperatura máxima $\left({ }^{\circ} \mathrm{C}\right)$, temperatura mínima $\left({ }^{\circ} \mathrm{C}\right)$, umidade relativa $(\%)$, velocidade média do vento $\left(\mathrm{m} \mathrm{s}^{-1}\right)$ e radiação solar global $\left(\mathrm{MJ} \mathrm{m}^{-2} \mathrm{dia}^{-1}\right)$.

$\mathrm{O}$ posto da ESALQ pertence ao Departamento de Engenharia de Biossistemas, localizada sob as coordenadas $22^{\circ} 42^{\prime} 30^{\prime \prime} \mathrm{S}$ e $47^{\circ} 38^{\prime} 00^{\prime \prime} \mathrm{W}$ (Piracicaba - SP), com altitude de 546 metros. O posto é composto de uma Estação Convencional e uma Estação Automática, que realiza observações meteorológicas a cada 15 minutos. Os dados para o estudo foram obtidos da Estação Automática que já converte os dados para uma base diária.

O clima de Piracicaba é considerado quente e temperado, com uma pluviosidade significativa ao longo do ano. O clima é classificado como Cfa segundo a Köppen e Geiger com temperatura média é $20,8{ }^{\circ} \mathrm{C}$ e pluviosidade média anual de $1.255 \mathrm{~mm}$.

O posto meteorológico do município baiano pertence ao Instituto Nacional de Meteorologia (INMET), localizada sob as coordenadas geográficas $12^{\circ} 09^{\prime} 00^{\prime \prime} \mathrm{S}$ e $45^{\circ}$
48’ 00” O (Luís Eduardo Magalhães - BA). O posto é constituído por estação automática com dados de base diária. O município de Luís Eduardo Magalhães apresenta clima tropical com chuvas concentradas no verão. O clima é classificado como Aw segundo a Köppen e Geiger com temperatura média anual de 24,2 ${ }^{\circ} \mathrm{C}$ e pluviosidade média anual é $1.511 \mathrm{~mm}$.

Foram utilizados dados diários compreendidos durante o período de janeiro de 2004 a dezembro de 2008. Conforme Garcia et al. (2017), não se utilizou dados com inconsistência de leitura ou registros incompletos, o que resultou numa amostra de 1.696 médias diárias viáveis para o município de Piracicaba - SP e 1.384 para o município de Luís Eduardo Magalhães - BA.

Após a seleção das variáveis climáticas, a evapotranspiração foi estimada por meio de cinco equações de diferentes modelos, sendo utilizado Penman-Monteith FAO56 (PMFAO56), Hargreaves-Samani (H-S), Radiação Solar FAO (RAD-FAO), Makkink (MKK) e Jensen-Haise (J-H) e em duas escalas de observação, uma anual, e outra sazonal, compreendendo as estações do ano (verão, outono, inverno e primavera). Dentre os modelos, PM-FAO56 foi definido como padrão e usado como referência para as comparações dos demais modelos.

\section{Método Penman-Monteith FAO56}

Como método padrão para estimativa da evapotranspiração de referência, utilizou-se o modelo proposto por Penman-Monteith (FAO56) (ALLEN et al., 1998), conforme Equação 1.

ETo $\frac{0,408 \Delta\left(R_{n}-G\right)+\gamma \frac{900}{T+273} \mathrm{U}_{2}\left(e_{s}-e_{a}\right)}{\Delta+\gamma\left(1+0,34 \mathrm{U}_{2}\right)}$

em que: $\mathrm{R}_{\mathrm{n}}$ - Radiação solar líquida, em MJ $\mathrm{m}^{-2} \mathrm{~s}^{-1} ; \mathrm{G}-$ Fluxo de calor no solo, em MJ $\mathrm{m}^{-2} \mathrm{~s}^{-1} ; \mathrm{T}-$ Temperatura média diária do ar, em ${ }^{\circ} \mathrm{C} ; \gamma$ - Constante psicrométrica, em kPa ${ }^{\circ} \mathrm{C}^{-1} ; \Delta$ - Tangente à curva de pressão de saturação de vapor em função da temperatura do ar, em $\mathrm{kPa}{ }^{\circ} \mathrm{C}^{-1} ; \mathrm{U}_{2}$ - Velocidade média diária do vento a 2 metros de altura, em $\mathrm{m} \mathrm{s}^{-1}$; $e_{a}-$ Pressão de vapor de saturação, média 
diária, em $\mathrm{kPa} ; e_{s}-$ Pressão de vapor de saturação, média diária, à temperatura do ponto de orvalho, em $\mathrm{kPa}$.

\section{Método Hargreaves-Samani}

A estimativa da evapotranspiração utilizando este método leva em consideração a diferença entre a temperatura máxima $\mathrm{e}$ mínima do ar, a temperatura média do ar e a radiação no topo da atmosfera como recomendado por Hargreaves e Samani (1985) na Equação 2.

$$
\text { ETo }=0,0023 \mathrm{R}_{\mathrm{a}} \sqrt{\mathrm{T}_{\text {max }}-\mathrm{T}_{\text {min }}}(\mathrm{T}+17,8)
$$

em que: $\mathrm{R}_{\mathrm{a}}$ - Radiação extraterrestre, em MJ $\mathrm{m}^{-2} \mathrm{dia}^{-1} ; \mathrm{T}_{\max }$ - Temperatura máxima do ar, em ${ }^{\circ} \mathrm{C} ; \mathrm{T}_{\min }$ - Temperatura mínima do ar, em ${ }^{\circ} \mathrm{C}$; $\mathrm{T}$ - Temperatura média do ar, em ${ }^{\circ} \mathrm{C}$.

\section{Método da Radiação Solar FAO}

Segundo Vescove e Turco (2005), o método da Radiação Solar FAO é uma adaptação feita por Doorenbos e Pruitt (1977) e Doorenbos e Kassam (1994) para o método Makkink, no qual substitui os coeficientes a e $\mathrm{b}$ do método por um parâmetro $\mathrm{c}$, que se relaciona com a umidade relativa do ar e a velocidade do vento conforme Equação 3.

$$
\mathrm{ETo}=\mathrm{c} \mathrm{W} \mathrm{R}
$$

em que: c - Coeficiente angular obtido pela Equação 4 conforme Doorenbos e Kassam (1979); W - Fator de ponderação dependente da temperatura do ar e coeficiente psicrométrico; $\mathrm{R}_{\mathrm{S}}$-Radiação solar ao nível da superfície do solo, expressa em equivalente de evaporação $\left(\mathrm{mm} \mathrm{dia}^{-1}\right)$.

$\mathrm{c}=\mathrm{c}_{0}+\mathrm{cL}$

em que: $c_{0}=-0,3 ; c L=a_{0}+a_{1} U R+a_{2} V d+$ $\mathrm{a}_{3} \mathrm{UR} \mathrm{Vd}+\mathrm{a}_{4} \mathrm{UR}^{2}+\mathrm{a}_{5} \mathrm{Vd}^{2}$ onde, $\mathrm{a}_{0}=$ 1,$0656 ; a_{1}=-0,0012795 ; a_{2}=0,044953 ; a_{3}=$ $-0,00020033 ; \quad a_{4}=-0,000031508 ; \quad a_{5}=$ -0,0011026; UR - Umidade relativa média, em \%; Vd - Velocidade média do vento no período diurno a $2 \mathrm{~m}$ de altura, em $\mathrm{m} \mathrm{s}^{-1}$, (considerou-se a $\mathrm{Vd}=$ a $70 \%$ da velocidade média do vento no período de 24 horas).
$\mathrm{O}$ fator $\mathrm{W}$ pode ser calculado seguindo metodologia proposta por Viswanadham et al. (1991), citada por Tanaka et al. (2016) de acordo com as Equações 5 e 6.

$$
\begin{aligned}
& \mathrm{W}=0,407+0,0145 \mathrm{~T} ; 0<\mathrm{T}<16,0{ }^{\circ} \mathrm{C} \\
& \mathrm{W}=0,483+0,01 \mathrm{~T} ; 16,1<\mathrm{T}<32{ }^{\circ} \mathrm{C}
\end{aligned}
$$

em que: $\mathrm{T}$ - Temperatura média do ar, em ${ }^{\circ} \mathrm{C}$.

\section{Método Makkink}

É um modelo simplificado ao padrão Penman Monteith FAO56, conforme Equação 7, onde Makkink substituiu o Saldo de Radiação pela Radiação Solar Global devido à dificuldade de se obter os dados de saldo de radiação, segundo Pereira et al. (1997).

$$
\mathrm{ETo}=0,61 \mathrm{~W} \mathrm{R}_{\mathrm{s}}-0,12
$$

em que: $R_{s}$ - Radiação solar ao nível da superfície do solo, expressa em equivalente de evaporação $\left(\mathrm{mm} \mathrm{dia}{ }^{-1}\right) ; \mathrm{W}-$ Fator de ponderação dependente da temperatura do ar e coeficiente psicrométrico.

\section{Método Jensen-Haise}

Segundo Pereira et al. (1997), é um modelo empírico desenvolvido para regiões semiáridas e áridas baseado na temperatura média do ar e na radiação solar global conforme descrito na Equação 8, segundo Ferronato et al. (2016).

$$
\mathrm{ETo}=(0,0252 \mathrm{~T}+0,078) \mathrm{R}_{\mathrm{s}}
$$

em que: $\mathrm{T}$ - Temperatura média do ar, em ${ }^{\circ} \mathrm{C}$; $\mathrm{R}_{\mathrm{s}}$ - Radiação solar ao nível da superfície do solo, expressa em equivalente de evaporação $\left(\mathrm{mm} \mathrm{dia}{ }^{-1}\right)$.

A comparação dos métodos analisados com o método padrão Penman Monteith FAO56 foi realizada por análise dos parâmetros de regressão linear $\beta_{1}$ e $\beta_{0}$, coeficiente de determinação $\left(\mathrm{R}^{2}\right)$, índice de concordância de Willmott (d) (WILLMOT et al., 1985), coeficiente de correlação (r) e confiança (c) (CAMARGO \& SENTELHAS, 1997) e raiz quadrada média do erro (RMSE), de acordo com as Equações 9, 10, 11 e 12 . 


$$
\mathrm{d}=1-\left[\frac{\sum_{\mathrm{i}=1}^{\mathrm{n}}(\mathrm{Pi}-\mathrm{Oi})^{2}}{\sum_{\mathrm{i}=1}^{\mathrm{n}}(|\mathrm{Pi}-\mathrm{O}|+|\mathrm{Oi}-\mathrm{O}|)^{2}}\right] \quad \text { (9) } \quad \text { RMSE }=\sqrt{\frac{\sum_{\mathrm{i}=1}^{\mathrm{n}}(\mathrm{Oi}-\mathrm{Pi})^{2}}{\mathrm{n}}}
$$$$
r=\frac{\sum_{i=1}^{n}(\mathrm{Pi}-\mathrm{P})(\mathrm{Oi}-\mathrm{O})}{\sqrt{\left[\sum_{\mathrm{i}=1}^{\mathrm{n}}(\mathrm{Pi}-\mathrm{P})^{2}\right]\left[\sum_{\mathrm{i}=1}^{\mathrm{n}}(\mathrm{Oi}-\mathrm{O})^{2}\right]}}
$$$$
\mathrm{c}=\mathrm{dr}
$$

em que: Oi - Evapotranspiração estimada pelo método padrão Penman-Monteith FAO56, em mm dia $^{-1} ; \mathrm{O}$ - média dos valores observados, em mm dia ${ }^{-1} ; \mathrm{Pi}$ - Evapotranspiração estimada pelo método a ser comparado, em $\mathrm{mm} \mathrm{dia}^{-1} ; \mathrm{P}$ - Média dos valores observados, em $\mathrm{mm} \mathrm{dia}^{-1}$; n - número de observações.

Para classificar o desempenho do método se utilizou a Tabela 1, segundo Camargo e Sentelhas (1997).

Tabela 1. Critérios para interpretação do índice de desempenho.

\begin{tabular}{cc}
\hline Índice de desempenho "c" & Classificação \\
\hline$>0,85$ & Excelente \\
$0,76-0,85$ & Muito Bom \\
$0,66-0,75$ & Bom \\
$0,61-0,65$ & Intermediário \\
$0,51-0,60$ & Tolerável \\
$0,41-0,50$ & Ruim \\
$\leq 0,4$ & Muito Ruim \\
\hline
\end{tabular}

Fonte: Camargo e Sentelhas (1997).

\section{RESULTADOS E DISCUSSÃO}

A Tabela 2 traz a média das variáveis meteorológicas e de evapotranspiração de referência $\left(\mathrm{ET}_{0}\right)$, estimada pelo método Penman Monteith FAO56, durante os cinco anos de estudo para os municípios de Luís Eduardo Magalhães - BA e Piracicaba - SP.

Tabela 2. Média das variáveis meteorológicas e evapotranspiração de referência $\left(\mathrm{ET}_{0}\right)$ estimada pelo

\begin{tabular}{|c|c|c|c|c|c|c|}
\hline \multirow{2}{*}{ Cidade } & \multicolumn{2}{|c|}{ Temperatura $\left({ }^{\circ} \mathrm{C}\right)$} & \multirow{2}{*}{$\begin{array}{c}\mathrm{U}_{2} \\
\left(\mathrm{~m} \mathrm{~s}^{-1}\right)\end{array}$} & \multirow{2}{*}{$\begin{array}{l}\text { UR } \\
(\%)\end{array}$} & \multirow{2}{*}{$\begin{array}{c}\text { RA } \\
\left(\mathrm{MJ} \mathrm{m}^{-2} \mathrm{dia}^{-1}\right)\end{array}$} & \multirow{2}{*}{$\begin{array}{c}\mathrm{ET}_{0} \\
\left(\mathrm{~mm} \mathrm{dia}^{-1}\right)\end{array}$} \\
\hline & Max & Min & & & & \\
\hline Piracicaba & 28,6 & 15,6 & 1,3 & 82,2 & 18,1 & 3,4 \\
\hline Luís Eduardo Magalhães & 30,8 & 17,0 & 2,9 & 61,7 & 23,9 & 5,4 \\
\hline
\end{tabular}
método Penman-Monteith FAO56, durante os anos de 2004 e 2008.

Max = Máxima; Min = Mínima; $\mathrm{U}_{2}=$ Velocidade do vento; $\mathrm{UR}=$ Umidade relativa do ar; RA = Radiação solar global; $\mathrm{ET}_{0}=$ Evapotranspiração de referência.

A tabela mostra que os valores de $\mathrm{ET}_{0}$ são mais elevados no estado baiano que no estado paulista, 5,4 e $3,4 \mathrm{~mm} \mathrm{dia}^{-1}$, respectivamente. Os valores são explicados devido à maior radiação solar global incidente no município baiano que se localiza mais próximo da linha do equador, onde a incidência da radiação solar é máxima (MARQUES et al., 2012) e, segundo Allen et al. (1998), a radiação solar é a principal variável climática que contribui para a evapotranspiração.

Os métodos Hargreaves-Samani e Jensen-Haise em Luís Eduardo Magalhães BA e Jensen-Haise em Piracicaba - SP tendem a superestimar os valores de $\mathrm{ET}_{0}$ (Figura 1). Tais resultados corroboram com resultados encontrados por Passos et al. (2017) em estudo que avaliaram o desempenho dos métodos de Camargo, Hargreaves-Samani, Jensen-Haise e 
Priestley-Taylor, na estimativa da $\mathrm{ET}_{0}$ diária, tendo como referência o método FAO Penman-Monteith, para o município de Chapadinha - MA onde, segundo o autor, os métodos Hargreaves-Samani, Jensen-Haise e Priestley-Taylor tiveram tendência em superestimar os valores de $\mathrm{ET}_{0}$.

No município de Luís Eduardo Magalhães, o método Hargreaves-Samani (H$\mathrm{S})$ apresentou valores de $\mathrm{ET}_{0}$ subestimados, demonstrando resultados de aproximadamente $87 \%$ dos valores de $\mathrm{ET}_{0}$ encontrados para o método FAO Penman-Monteith. Porém, ao analisar a média anual da evapotranspiração de referência na mesorregião de Piracicaba, nota- se que H-S demonstrou valores superestimados em relação ao método padrão.

De acordo com a Figura 1, observa-se que os métodos Makkink (MKK) e Radiação Solar FAO (RAD) tendem a subestimar os valores, tanto para o município baiano como para o paulista. Ao analisar séries históricas para determinação de $\mathrm{ET}_{0}$ dos municípios brasileiros de Aimorés (MG) e Linhares (ES), Garcia et al. (2019) observaram, assim como neste trabalho, a subestimativa para o método MKK e contrariamente, valores superestimados para o método RAD.
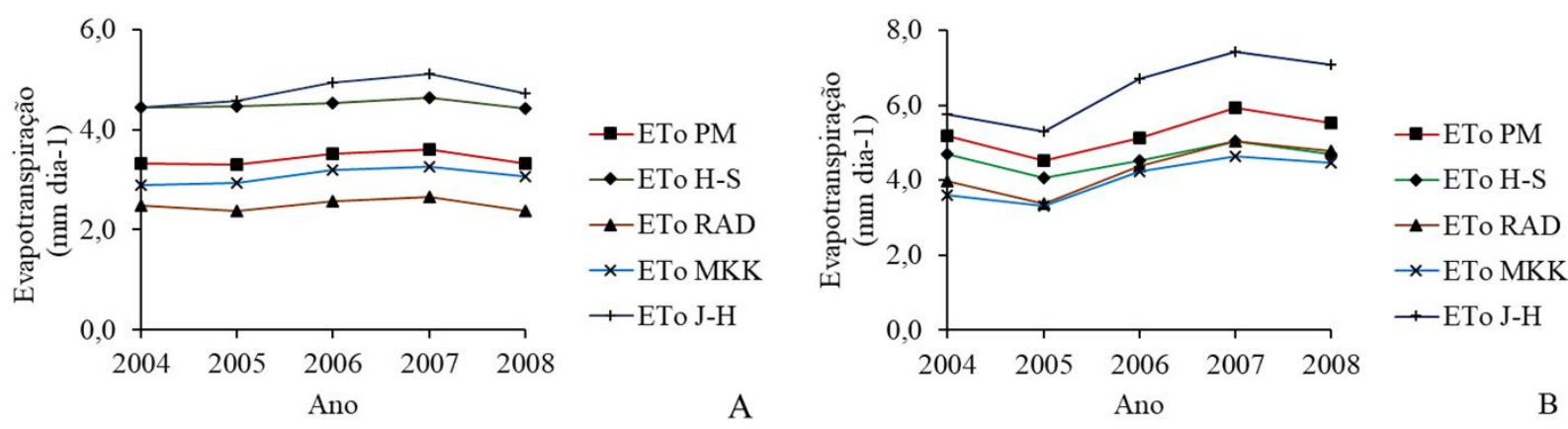

Figura 1. Média anual da estimativa de $\mathrm{ET}_{0}$ para os diferentes métodos para as localidades de Piracicaba - SP (A) e Luís Eduardo Magalhães - BA (B). Fonte: autores.

Figura 2. Média anual da estimativa de $\mathrm{ET}_{0}$ para os diferentes métodos para as localidades de Piracicaba - SP (A) e Luís Eduardo Magalhães - BA (B). Fonte: autores.

Os parâmetros $\beta_{1}$ e $\beta_{0}$ da regressão linear, bem como o coeficiente de determinação $\mathrm{R}^{2}$, para cada método avaliado em comparação ao método padrão Penman
Monteith FAO56, em escala anual, encontrase na Tabelas 3 e as Figuras $2 \mathrm{~A}$ e $2 \mathrm{~B}$ trazem a representação gráfica das regressões.

Tabela 3. Parâmetros da regressão linear da comparação de cada método para os dois municípios em escala anual.

\begin{tabular}{clccc}
\hline \multirow{2}{*}{ Cidade } & \multicolumn{1}{c}{ Método } & \multicolumn{3}{c}{ Coeficiente de Regressão } \\
\cline { 3 - 4 } & Hargreaves-Samani & 0,9227 & $\beta 0$ & $\mathrm{R}^{\mathbf{2}}$ \\
\hline \multirow{2}{*}{ Piracicaba } & 0,7674 & 1,3530 & 0,8121 \\
& Radiação Solar FAO & 0,8213 & $-0,1249$ & 0,8787 \\
& Makkink & 1,4349 & 0,2604 & 0,9437 \\
& Jensen-Haise & 0,5178 & $-0,1381$ & 0,9636 \\
\hline \multirow{2}{*}{ Luís Eduardo } & Hargreaves-Samani & 0,9169 & 1,8932 & 0,4727 \\
Magalhães & Radiação Solar FAO & 0,6062 & $-0,4609$ & 0,8678 \\
& Makkink & 0,9202 & 0,7222 \\
& Jensen-Haise & 1,0316 & 1,1051 & 0,7552 \\
\hline
\end{tabular}

O menor valor do coeficiente de determinação $\mathrm{R}^{2}$, em escala anual, foi observado na comparação entre o método padrão PM-FAO56 e H-S, assumindo valores 


\section{COMPARAÇÃO ENTRE MÉTODOS DE ESTIMATIVA DA EVAPOTRANSPIRAÇÃO DE REFERÊNCIA NO OESTE BAIANO E MESORREGIÃO PAULISTA}

de 0,8121 e 0,4727 para Piracicaba - SP e Luís Eduardo Magalhães - BA, respectivamente. A mesma comparação apresentou o pior ajuste devido aos valores de $\beta_{1}$ e $\beta_{0}$ onde, segundo Venâncio et al. (2019), um bom ajuste de modelos apresenta valores de $\beta_{0}$ próximos de zero e valores de $\beta_{1}$ próximos de 1.

Este fato pode ser observado uma vez que o coeficiente angular $\beta_{1}$ faz variar em proporções múltiplas, enquanto o valor de $\beta_{0}$ (coeficiente linear) atua como uma constante para ajustar os valores estimados ao padrão. Portanto, quando o coeficiente $\beta_{1}$ é igual a 1 não há grandes variações, pois múltiplos de 1 não se alteram, enquanto para os valores de $\beta_{0}$ mais próximos de 0 não há grandes acréscimos ou subtrações de constante, ou seja, o valor estimado se aproxima do real.

Já os maiores valores de $\mathrm{R}^{2}$, em escala anual, foram observados na comparação entre PM-FAO56 e J-H em Piracicaba - SP, 0,9636, e PM-FAO56xRAD em Luís Eduardo Magalhães - BA, 0,8678.
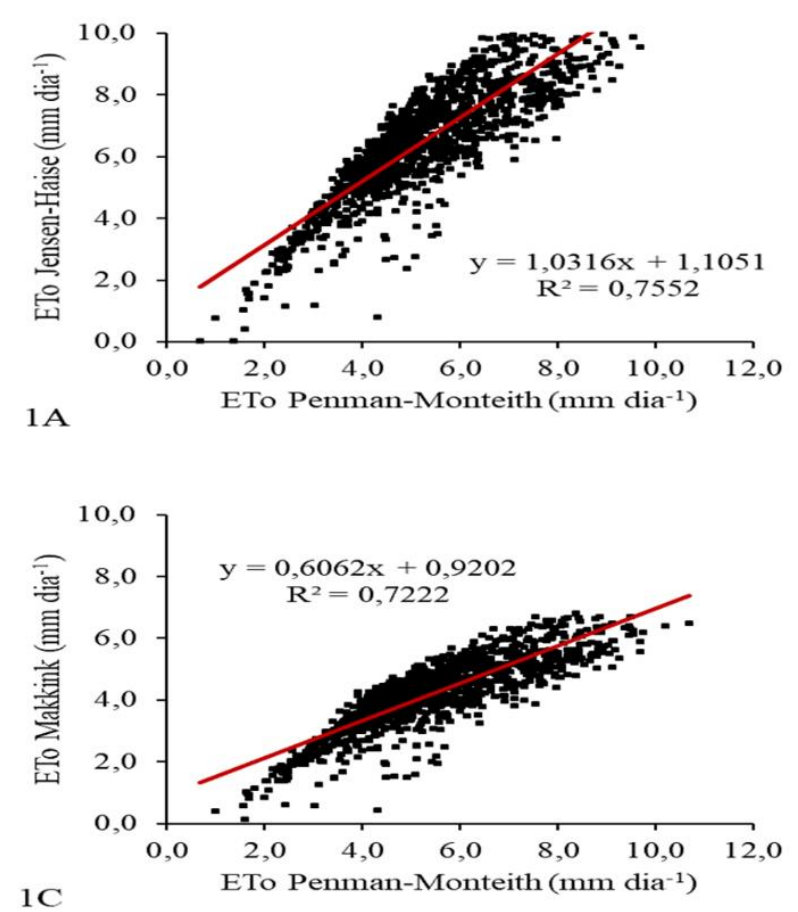

Para o município de Piracicaba nota-se que os valores de $\mathrm{R}^{2}$ se mantiveram com pouca alteração independente da comparação em escala anual ou sazonal e com os valores mais elevados oscilando entre os métodos $\mathrm{J}-\mathrm{H}$ e MKK. No município Baiano a comparação sazonal resultou em elevação dos valores de correlação e verifica-se que a maior correlação, em todas as estações, é observada pelo método RAD-FAO, com correlações iguais ou superiores a $0,7461 \mathrm{e}$ as menores correlações são observadas no método $\mathrm{H}-\mathrm{S}$ com valores inferiores a 0,6704. Cabral Júnior et al. (2017), estudando a comparação mensal, sazonal e anual de métodos de estimativas da evapotranspiração de referência para JuazeiroBA e Petrolina-PE obtiveram resultados similares onde, em escala sazonal, a maior correlação foi observada para o método de Radiação Solar, com valores superiores a 0,93, e as menores correlações oscilaram entre o método Tanque Classe A e HargreavesSamani.
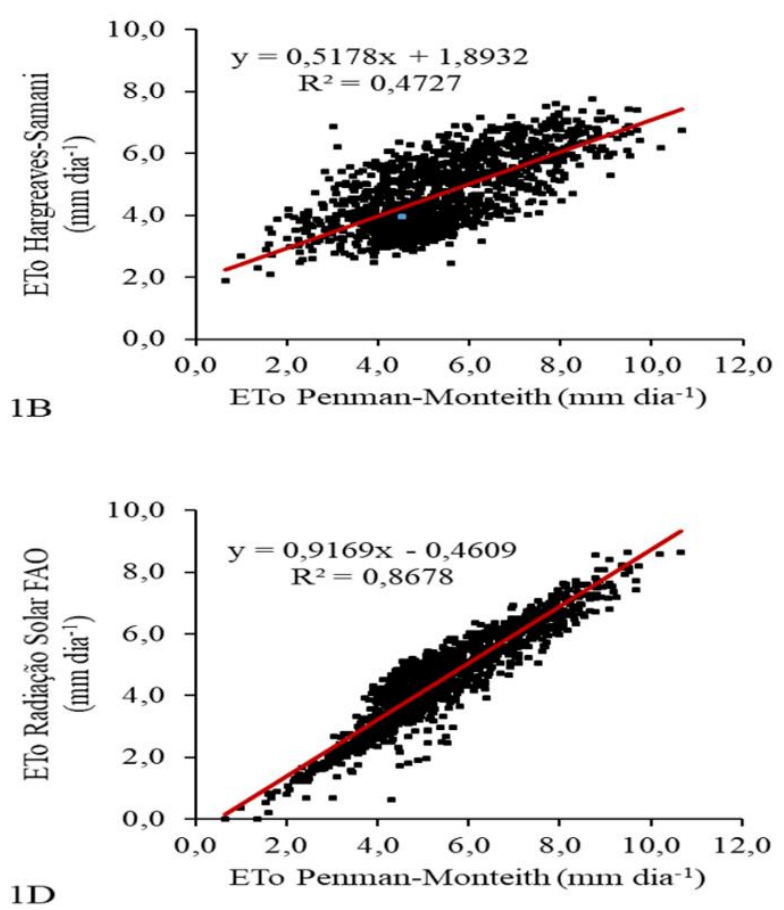

Figura 3A. Representação gráfica das regressões lineares da comparação dos métodos J-H (1A), H-S (1B), MKK (1C) e RAD (1D) para o município de Luís Eduardo Magalhães, em escala anual. Fonte: autores. 

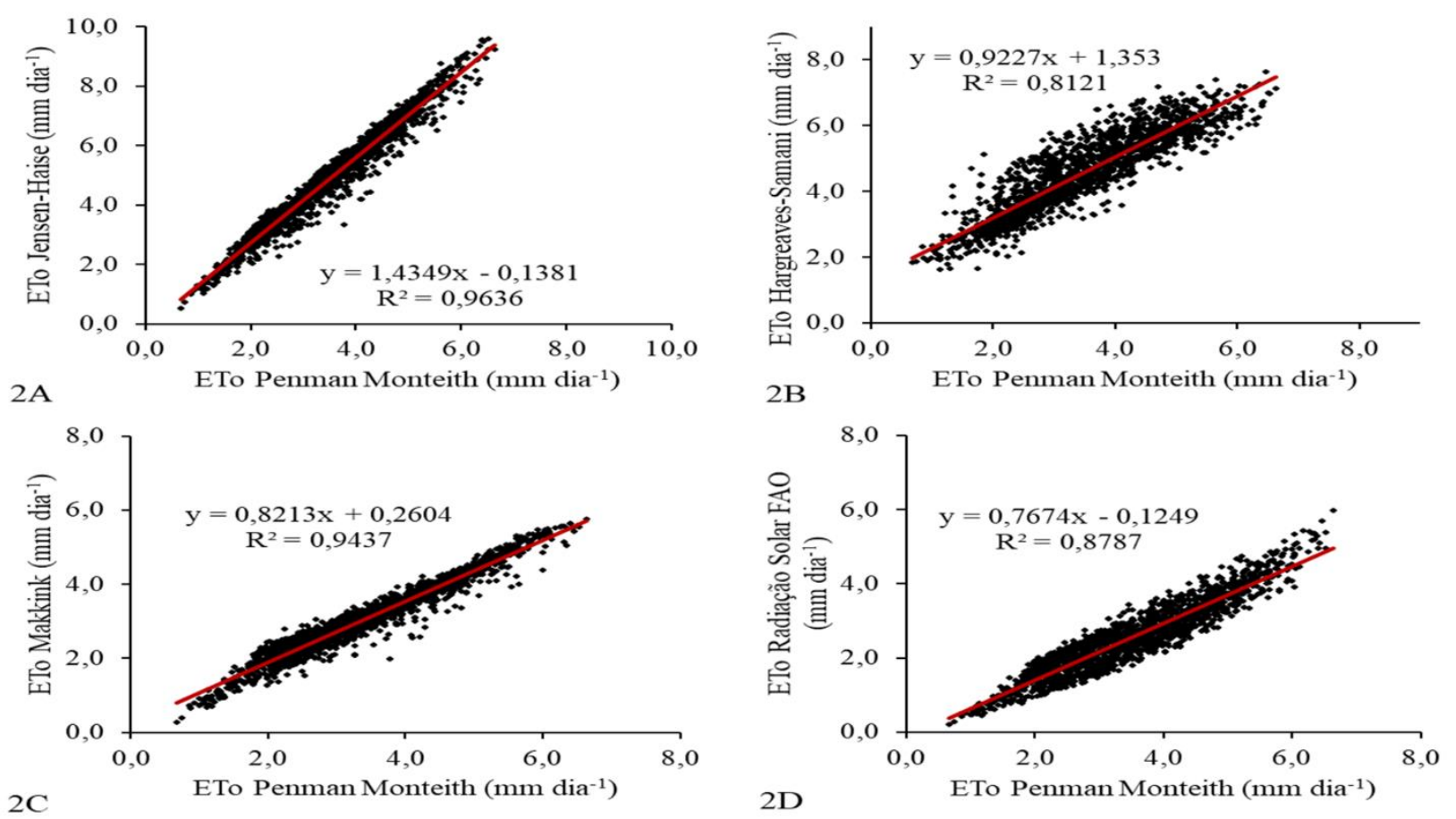

Figura 2B. Representação gráfica das regressões lineares da comparação dos métodos J-H (1A), H-S (1B), MKK (1C) e RAD (1D) para o município de Piracicaba J-H (2A), H-S (2B), MKK (2C) e RAD (2D), em escala anual. Fonte: autores.

A Tabela 4 apresenta $\beta_{1}, \beta_{0}$, e $\mathrm{R}^{2}$ para cada método avaliado em comparação ao método padrão Penman-Monteith FAO56, em escala sazonal. Observou-se que os dois menores valores de $\mathrm{R}^{2}$ encontrados em cada município foram estimados pelo método Hargreaves-Samani: em Piracicaba, 0,6936 e 0,6498, para estação do verão e primavera, respectivamente e; no oeste baiano, 0,3393 e 0,6188 , para estação do outono e primavera, nesta ordem.

Nota-se para o município baiano que o método $\mathrm{H}-\mathrm{S}$ apresentou os maiores valores de $\mathrm{R}^{2}$ na estação do verão, isso se dá devido ao fato de que este se trata de um método empírico desenvolvido para uso em regiões semiáridas (Hargreaves e Samani, 1985) e para região estudada, esta é a estação que mais se parece com tal condição. Resultados similares foram encontrados por Venâncio et al. (2019), em que o método H-S apresentou maior eficiência no período de verão se comparado às demais estações do ano.

Entretanto, Lima Júnior et al. (2016) observaram que o método Hargreaves- Samani tende a superestimar a ET0 nos meses inciais do ano no estado do Ceará, com clima semiárido, mesmo após a calibração deste método com o PM-FAO56, pois este período apresentou maiores quantidades de chuvas e menores térmicas. 

REFERÊNCIA NO OESTE BAIANO E MESORREGIÃO PAULISTA

Tabela 4. Parâmetros da regressão linear da comparação de cada método para os dois municípios em escala sazonal.

\begin{tabular}{|c|c|c|c|c|c|}
\hline \multirow{2}{*}{ Cidade } & \multirow{2}{*}{ Estação do ano } & \multirow{2}{*}{ Método } & \multicolumn{3}{|c|}{ Coeficientes da regressão } \\
\hline & & & $\beta 1$ & $\beta 0$ & $\mathrm{R}^{2}$ \\
\hline \multirow{16}{*}{ Piracicaba } & \multirow{4}{*}{ Verão } & Hargreaves-Samani & 0,5826 & 2,9570 & 0,6936 \\
\hline & & Radiação Solar FAO & 0,9121 & $-0,9998$ & 0,9421 \\
\hline & & Makkink & 0,9270 & $-0,2206$ & 0,9808 \\
\hline & & Jensen-Haise & 1,5324 & $-0,4408$ & 0,9743 \\
\hline & \multirow{4}{*}{ Outono } & Hargreaves-Samani & 0,8913 & 1,1920 & 0,8300 \\
\hline & & Radiação Solar FAO & 0,7513 & $-0,0193$ & 0,8628 \\
\hline & & Makkink & 0,8766 & 0,2148 & 0,9146 \\
\hline & & Jensen-Haise & 1,5458 & $-0,2662$ & 0,9668 \\
\hline & \multirow{4}{*}{ Inverno } & Hargreaves-Samani & 0,9462 & 1,0775 & 0,8338 \\
\hline & & Radiação Solar FAO & 0,8852 & $-0,1950$ & 0,9304 \\
\hline & & Makkink & 0,7304 & 0,5301 & 0,8571 \\
\hline & & Jensen-Haise & 1,2552 & 0,2345 & 0,9203 \\
\hline & \multirow{4}{*}{ Primavera } & Hargreaves-Samani & 0,6650 & 2,7126 & 0,6498 \\
\hline & & Radiação Solar FAO & 0,9690 & $-1,0303$ & 0,9370 \\
\hline & & Makkink & 0,9065 & $-0,1759$ & 0,9509 \\
\hline & & Jensen-Haise & 1,4598 & $-0,3820$ & 0,9617 \\
\hline \multirow{16}{*}{$\begin{array}{l}\text { Luís Eduardo } \\
\text { Magalhães }\end{array}$} & \multirow{4}{*}{ Verão } & Hargreaves-Samani & 0,4991 & 2,8185 & 0,6704 \\
\hline & & Radiação Solar FAO & 1,0056 & $-1,2605$ & 0,9632 \\
\hline & & Makkink & 0,8191 & $-0,1053$ & 0,8516 \\
\hline & & Jensen-Haise & 1,3568 & $-0,2787$ & 0,8770 \\
\hline & \multirow{4}{*}{ Outono } & Hargreaves-Samani & 0,3789 & 2,0809 & 0,3393 \\
\hline & & Radiação Solar FAO & 1,1053 & $-1,0778$ & 0,8677 \\
\hline & & Makkink & 0,9155 & $-0,2106$ & 0,8217 \\
\hline & & Jensen-Haise & 1,4451 & $-0,3471$ & 0,8246 \\
\hline & \multirow{4}{*}{ Inverno } & Hargreaves-Samani & 0,5861 & 0,8170 & 0,6583 \\
\hline & & Radiação Solar FAO & 0,7585 & 0,7818 & 0,7461 \\
\hline & & Makkink & 0,4543 & 1,7221 & 0,5425 \\
\hline & & Jensen-Haise & 0,8289 & 1,8407 & 0,6300 \\
\hline & \multirow{4}{*}{ Primavera } & Hargreaves-Samani & 0,3749 & 3,4939 & 0,6188 \\
\hline & & Radiação Solar FAO & 0,9924 & $-1,3292$ & 0,9563 \\
\hline & & Makkink & 0,6411 & 0,4514 & 0,7901 \\
\hline & & Jensen-Haise & 1,0903 & 0,5802 & 0,8086 \\
\hline
\end{tabular}

Os coeficientes de comparação da raiz quadrada média do erro (RMSE), índice de concordância (d), índice de correlação (r) e de confiança (c) para cada método avaliado em comparação ao método padrão, em escala anual e sazonal, encontram-se nas Tabelas 5 e 6.

De acordo com as Tabelas 5 e 6 , os resultados encontrados para a classificação de "c" descrevem o método MKK como excelente, tanto na escala sazonal como na anual para a mesorregião de Piracicaba, com valores superiores a 0,93 e baixa raiz quadrada média do erro de estimativa, com valores abaixo de $0,6 \mathrm{~mm}$ dia- 1 . Tal resultado diverge do encontrado por Tangune (2017), onde o método MKK apresentou o menor desempenho com valor de RMSE igual a 1,43 $\mathrm{mm}$ dia-1. Observa-se para o município de Piracicaba - SP, que os métodos $\mathrm{H}-\mathrm{S}$ e J-H são os que apresentam maiores valores de RMSE (mm dia-1), em escala anual e sazonal, esses valores elevados são explicados devido à tendência destes métodos em superestimar a ET0 em comparação com o método padrão (PASSOS et al., 2017). 
Nandorf et al.

Tabela 5. Coeficientes de comparação de cada método para os dois municípios em escala anual.

\begin{tabular}{|c|c|c|c|c|c|c|}
\hline \multirow[b]{2}{*}{ Cidade } & \multirow[b]{2}{*}{ Método } & \multicolumn{5}{|c|}{ Coeficientes de comparação } \\
\hline & & $\begin{array}{c}\text { RMSE (mm } \\
\left.\mathrm{dia}^{-1}\right)\end{array}$ & d & $\mathrm{r}$ & $\mathrm{c}$ & Classificação de c \\
\hline \multirow{4}{*}{ Piracicaba } & Hargreaves-Samani & 1,2 & 0,79 & 0,90 & 0,71 & Bom \\
\hline & Radiação Solar FAO & 1,0 & 0,82 & 0,94 & 0,77 & Muito Bom \\
\hline & Makkink & 0,5 & 0,95 & 0,97 & 0,93 & Excelente \\
\hline & Jensen-Haise & 1,5 & 0,79 & 0,98 & 0,78 & Muito Bom \\
\hline \multirow{4}{*}{$\begin{array}{c}\text { Luís Eduardo } \\
\text { Magalhães }\end{array}$} & Hargreaves-Samani & 1,3 & 0,76 & 0,69 & 0,52 & Tolerável \\
\hline & Radiação Solar FAO & 1,1 & 0,89 & 0,93 & 0,83 & Muito Bom \\
\hline & Makkink & 1,5 & 0,23 & 0,85 & 0,20 & Muito Ruim \\
\hline & Jensen-Haise & 1,6 & 0,81 & 0,87 & 0,71 & Bom \\
\hline
\end{tabular}

Observa-se para o município de Piracicaba - SP, que os métodos H-S e J-H são os que apresentam maiores valores de RMSE (mm dia ${ }^{-1}$ ), em escala anual e sazonal, esses valores elevados são explicados devido à tendência destes métodos em superestimar a $\mathrm{ET}_{0}$ em comparação com o método padrão (PASSOS et al., 2017).

A raiz quadrada do erro médio ou RMSE indica, em um mesmo padrão positivo, os erros médios obtidos pelos métodos, ou seja, quanto maior o valor de RMSE maior também será o erro gerado pelo modelo. Além disso, quanto maior o erro médio absoluto, maior também será a RSME, pois esse índice racionaliza o erro a segunda potência.

Os resultados apresentados nas Tabelas 5 e 6 indicam que o método HargreavesSamani não é recomendado para estimativa da evapotranspiração de referência $\left(\mathrm{ET}_{0}\right)$ em Luís Eduardo Magalhães, pois tanto na escala anual como nas estações de outono, inverno e primavera os valores de "c" estiveram abaixo de 0,59, sendo classificado como tolerável. Resultados análogos foram observados por Cabral Júnior et al. (2017), avaliando do desempenho de métodos para estimativa de $\mathrm{ET}_{0}$ diária, com valores de "c" iguais a $0,10 \mathrm{e}$ 0,02 para Juazeiro - BA e Petrolina - PE, respectivamente.

Os valores de "c" obtidos para análise anual dos dados meteorológicos da mesorregião de Piracicaba são superiores a 0,70, sendo classificados entre "bom" e "excelente", assim como resultados encontrados por Brixner et al. (2014), em que os métodos RAD e MKK apresentaram valores e classificação similares na Campanha Gaúcha (RS). Entretanto, na análise sazonal, somente as estações verão e primavera apresentam valores similares e possuem as mesmas classificações para todos os ajustes, onde os métodos H-S, RAD, MKK e J-H são classificados como "tolerável", "bom", "excelente" e "bom", respectivamente. As estações inverno e outono não apresentaram classificações semelhantes.

O município de Luís Eduardo Magalhães apresenta como destaque na escala anual o método Radiação Solar FAO, com coeficiente de confiança classificado como "muito bom". Este resultado se deve ao fato de que o método da Radiação Solar FAO tem maior eficiência em regiões próximas a linha do equador, pequenas ilhas ou em grandes altitudes, conforme indicam Doorenbos e Pruitt (1977), onde há maior incidência de radiação durante o dia, que é a principal variável de entrada desta metodologia.

Contrariamente, os métodos que apresentaram resultados inferiores e classificam-se com "muito ruim" e "tolerável", são Makkink e Hargreaves-Samani, logo, não são recomendados para esta região. Assim como na escala anual, o método RAD apresenta-se como mais adequado para estimativas de ET0 nas quatro estações do ano para o oeste baiano, com valores de "c" estimados entre 0,74 e 0,85 , sendo classificado como "muito bom". 
COMPARAÇÃO ENTRE MÉTODOS DE ESTIMATIVA DA EVAPOTRANSPIRAÇÃO DE REFERÊNCIA NO OESTE BAIANO E MESORREGIÃO PAULISTA

Tabela 6. Coeficientes de comparação de cada método para os dois municípios em escala sazonal.

\begin{tabular}{|c|c|c|c|c|c|c|c|}
\hline \multirow[b]{2}{*}{ Cidade } & \multirow{2}{*}{$\begin{array}{l}\text { Estação do } \\
\text { ano }\end{array}$} & \multirow[b]{2}{*}{ Método } & \multicolumn{5}{|c|}{ Coeficientes de comparação } \\
\hline & & & $\begin{array}{c}\text { RMSE (mm } \\
\left.\mathrm{dia}^{-1}\right)\end{array}$ & d & $\mathrm{r}$ & $\mathrm{c}$ & Classificação de c \\
\hline \multirow{16}{*}{ Piracicaba } & \multirow{4}{*}{ Verão } & $\mathrm{H}-\mathrm{S}$ & 1,4 & 0,65 & 0,83 & 0,54 & Tolerável \\
\hline & & RAD & 1,4 & 0,72 & 0,97 & 0,70 & Bom \\
\hline & & MKK & 0,6 & 0,94 & 0,99 & 0,93 & Excelente \\
\hline & & $\mathrm{J}-\mathrm{H}$ & 1,9 & 0,68 & 0,99 & 0,67 & Bom \\
\hline & \multirow{4}{*}{ Outono } & $\mathrm{H}-\mathrm{S}$ & 1,0 & 0,68 & 0,91 & 0,62 & Intermediário \\
\hline & & RAD & 0,7 & 0,30 & 0,93 & 0,28 & Muito Ruim \\
\hline & & MKK & 0,3 & 0,94 & 0,96 & 0,90 & Excelente \\
\hline & & $\mathrm{J}-\mathrm{H}$ & 1,3 & 0,66 & 0,98 & 0,65 & Intermediário \\
\hline & \multirow{4}{*}{ Inverno } & $\mathrm{H}-\mathrm{S}$ & 1,0 & 0,76 & 0,91 & 0,69 & Bom \\
\hline & & RAD & 0,6 & 0,89 & 0,96 & 0,86 & Excelente \\
\hline & & MKK & 0,4 & 0,93 & 0,93 & 0,86 & Excelente \\
\hline & & $\mathrm{J}-\mathrm{H}$ & 1,0 & 0,79 & 0,96 & 0,76 & Muito Bom \\
\hline & \multirow{4}{*}{ Primavera } & $\mathrm{H}-\mathrm{S}$ & 1,5 & 0,64 & 0,81 & 0,52 & Tolerável \\
\hline & & RAD & 1,2 & 0,77 & 0,97 & 0,75 & Bom \\
\hline & & MKK & 0,6 & 0,92 & 0,98 & 0,90 & Excelente \\
\hline & & $\mathrm{J}-\mathrm{H}$ & 1,6 & 0,73 & 0,98 & 0,71 & Bom \\
\hline \multirow{16}{*}{$\begin{array}{c}\text { Luís } \\
\text { Eduardo } \\
\text { Magalhães }\end{array}$} & \multirow{4}{*}{ Verão } & $\mathrm{H}-\mathrm{S}$ & 1,0 & 0,84 & 0,82 & 0,68 & Bom \\
\hline & & RAD & 1,3 & 0,85 & 0,98 & 0,84 & Muito Bom \\
\hline & & MKK & 1,2 & 0,85 & 0,92 & 0,78 & Muito Bom \\
\hline & & $\mathrm{J}-\mathrm{H}$ & 1,8 & 0,80 & 0,94 & 0,75 & Bom \\
\hline & \multirow{4}{*}{ Outono } & $\mathrm{H}-\mathrm{S}$ & 1,0 & 0,63 & 0,58 & 0,37 & Muito Ruim \\
\hline & & RAD & 0,7 & 0,88 & 0,93 & 0,82 & Muito Bom \\
\hline & & MKK & 0,7 & 0,86 & 0,91 & 0,78 & Muito Bom \\
\hline & & $\mathrm{J}-\mathrm{H}$ & 1,8 & 0,63 & 0,91 & 0,57 & Tolerável \\
\hline & \multirow{4}{*}{ Inverno } & $\mathrm{H}-\mathrm{S}$ & 1,7 & 0,61 & 0,81 & 0,49 & Ruim \\
\hline & & RAD & 0,8 & 0,86 & 0,86 & 0,74 & Bom \\
\hline & & MKK & 1,6 & 0,60 & 0,74 & 0,44 & Ruim \\
\hline & & $\mathrm{J}-\mathrm{H}$ & 1,1 & 0,79 & 0,79 & 0,63 & Intermediário \\
\hline & \multirow{4}{*}{ Primavera } & $\mathrm{H}-\mathrm{S}$ & 1,4 & 0,75 & 0,79 & 0,59 & Tolerável \\
\hline & & RAD & 1,4 & 0,87 & 0,98 & 0,85 & Muito Bom \\
\hline & & MKK & 2,0 & 0,72 & 0,89 & 0,64 & Intermediário \\
\hline & & $\mathrm{J}-\mathrm{H}$ & 1,5 & 0,87 & 0,90 & 0,78 & Muito Bom \\
\hline
\end{tabular}

Embora o método de HargreavesSamani seja o menos recomendado para a estimativa da $\mathrm{ET}_{0}$ para o oeste baiano, nota-se algumas vantagens, especialmente pela sua simplicidade em relação aos outros métodos, já que este necessita somente de dados de temperatura, facilmente adquiridos com um termômetro. Em contraposição, os outros métodos carecem de estações meteorológicas que informem dados de radiação solar, crucial para estimativa da evapotranspiração de referência. Neste contexto, nota-se que em propriedades rurais e pesquisas agrícolas situadas em áreas que não possuem estações meteorológicas próximas, o método $\mathrm{H}-\mathrm{S}$ tornase usual e prático, já que os outros métodos de estimativa tornariam o processo oneroso pela presença obrigatória de infraestrutura agrometeorológica.

\section{CONCLUSÕES}

O método Makkink apresenta-se como o mais recomendado para a mesorregião de Piracicaba, tanto em escala anual como sazonal, enquanto para Luís Eduardo Magalhães, o método Radiação Solar FAO torna-se o mais apropriado para estimativa da ET $_{0}$ tanto em escala anual quanto sazonal. Dentre os métodos analisados, HargreavesSamani torna-se o menos recomendado para estimativa da Evapotranspiração de Referência $\left(\mathrm{ET}_{0}\right)$ em Luís Eduardo Magalhães. Já em 
Piracicaba, o método Hargreaves-Samani pode ser utilizado, principalmente pela sua simplicidade.

\section{REFERÊNCIAS BIBLIOGRÁFICAS}

A.I.B.A. Irrigar para crescer. In: CONGRESSO NACIONAL DE IRRIGAÇÃO E DRENAGEM, 13., 2013, Luís Eduardo Magalhães. Anais eletrônicos... Luís Eduardo Magalhães: CONIRD, 2013. Disponível em: <http://www.aiba.org.br/noticia/4341/xxiiiconirdirrigar-para-crescer/ $>$. Acesso em: 03 abr. 2020.

ALLEN, R. G.; PEREIRA, L. S.; RAES, D.; SMITH, M. Crop evapotranspiration: Guidelines for computing crop water requirements. Roma: FAO, 1998. (FAO Irrigation and Drainage Paper n. 56.)

ANTONOPOULOS, $\quad$ V. Z.; ANTONOPOULOS, A. V. Daily reference evapotranspiration estimates by artificial neurais networks technique and empirical equations using limited input climate variables. Computers and Electronics in Agriculture, v.132, p.86 - 96, 2017. https://doi.org/10.1016/j.compag.2016.11.011

BRIXNER, G. F. SCHOFFEL, E. R.; TONIETTO, J. Determinação da evapotranspiração por diferentes métodos e sua aplicação no índice de seca na Campanha Gaúcha, Brasil. Revista Brasileira de Fruticultura, v. 36, n. 4, p. 780-793, 2014. https://doi.org/10.1590/0100-2945-381/13

CABRAL JÚNIOR, J. B.; SILVA, C. M. S.; ALMEIDA, H. A. Comparação mensal, sazonal e anual de métodos de estimativas da evapotranspiração de referência para JuazeiroBA e Petrolina-PE. Revista de Geociências do Nordeste. v. 3, n. 2, p. 23-42, 2017.

CAMARGO, A. P.; SENTElHAS, P. C. Avaliação do desempenho de diferentes métodos de estimativa da evapotranspiração potencial no estado de São Paulo, Brasil. Revista Brasileira de Agrometeorologia, v.5, n.1, p.89-97, 1997.

DOORENBOS, J.; KASSAM, A.H. Crop evapotranspiration: Yield response to water. Rome: FAO, 1979. (FAO Irrigation and Drainage Paper n. 33).

DOORENBOS, J.; PRUITT, W.O. Crop evapotranspiration: Guidelines for predicting crop water requirements. Rome: FAO, 1977. (Irrigation and Drainage Paper n. 24).

FERRONATO A.; CHIG, L. A.; GOULART, D. B.; JÚNIOR, J. H. C.; PEREIRA, L. C.; BIUDES, M. S. Métodos de estimativa da evapotranspiração de referência para Santo Antônio do Leverger-MT. Revista de Ciências Agroambientais, v.14, n.1, p.110118, 2016.

FOOD AND AGRICULTURE ORGANIZATION OF THE UNITED NATIONS - FAO. Information system on water and agriculture - AQUASTAT. Rome: FAO, 2016. Disponível em: $<$ http://www.fao.org/nr/water/aquastat/water_ use/index.stm>. Acesso em: 09 mar. 2020.

GARCIA, A. D. B.; MENDONÇA, J. C.; ALMEIDA, C. M. Métodos de estimativa da evapotranspiração de referência $\left(\mathrm{ET}_{0}\right)$ no município de Santa Teresa, ES. Irriga, v. 22, n. $\quad 4, \quad$ p. $701-714, \quad 2017$. https://doi.org/10.15809/irriga.2017v22n4p70 $1-714$

GARCIA, A. D. B.; VIEIRA, G. H. S.; PERTELE, G. BONADIMAN, P. A.; FRIZZERA, J. L.; GRAZZIOTI, R. M.; LO MONACO, P. A. V.; HADDADE, I. R.; CHAMBELA NETO, A. Options for Reference Evapotranspiration Estimation in Northern Espírito Santo and Eastern Minas Gerais States, Brazil. Journal of Experimental Agriculture International, v. 29, n. 2, p. $1-14,2019$. https://doi.org/10.9734/JEAI/2019/45818 
HARGREAVES, G. H.; SAMANI, Z. A. Reference crop evapotranspiration from temperature. Applied Engineering in Agriculture, v. 01, n. 02, p. 96-99, 1985.

LIMA JÚNIOR, J. C.; ARRAES, F. D. D.; OLIVEIRA, J. B.; NASCIMENTO, F. A. L.; MACEDO, K. G. Parametrização da equação de Hargreaves e Samani para estimativa de evapotranspiração de referência no estado do Ceará, Brasil. Revista Ciência Agronômica, v.47, n.3, p.447-454, 2016. https://doi.org/10.5935/1806-6690.20160054

MARQUES, D. D.; BRITO, A. U.; CUNHA, A. C. SOUZA, L. R. Variação da radiação solar no estado do amapá: estudo de caso em Macapá, Picuí, Serra do Navio e Oiapoque no período de 2006 a 2008. Revista Brasileira de Meteorologia, v.27, n.2, 127 - 138, 2012. https://doi.org/10.1590/S0102-

77862012000200002

MONTEIRO, L. A. Modelagem agrometereológica com base para a definição de ambientes de produção apara acultura da cana-de-açúcar no Estado de São Paulo. 2012. 116f. Dissertação (Mestrado em Ciências) - Escola Superior de Agricultura "Luiz de Queiroz", Piracicaba.

PASSOS, M. L. V.; RAPOSO, A. B.; MENDES, T. J. Evapotranspiração de referência por diferentes métodos para o município de Chapadinha-MA. Revista Agropecuária Cientifica no Semiárido, v.13, n.2, p.146-153, 2017.

PAULINO, V. E. N.; STUDART, T. M. C. CAMPOS, J. N. B.; PESTANA, C. J.; LUNA, R. M.; ALVES. J. M. B. Análise de Tendências na Evapotranspiração de Referência e nas Variáveis Climatológicas do Estado do Ceará-Brasil. Revista Brasileira de Meteorologia, v.34, n.1, p.79-88, 2019. https://doi.org/10.1590/0102-7786334017.

PEREIRA, A. R.; VILLA NOVA, N. A.; SEDIYAMA, G. C. Evapo(transpi)ração. Piracicaba: FEALQ, 1997. 183p.
TANAKA, A. A.; DE SOUZA, A. P.; KLAR, A. E.; DA SILVA, A. C.; GOMES, A. W. A. Evapotranspiração de referência estimada por modelos simplificados para o Estado do Mato Grosso. Pesquisa Agropecuária Brasileira, v. 51, n. 2, p. 91-104, 2016. https://doi.org/10.1590/S0100-

204X2016000200001

TANGUNE，B. F. Evapotranspiração de referência no estado de São Paulo: métodos empíricos, aprendizado de máquina geoespacial. 2017. 126f. Tese (Doutorado em Agronomia). Universidade Estadual Paulista, Faculdade de Ciências Agronômicas, Botucatu.

VENÂNCIO, L. P.; CUNHA, F. F.; MANTOVANI, E. C.; SEDIYAMA, G. C.; EUGENIO, F. C.; ALEMAN, C. C. PenmanMonteith com dados faltantes e HargreavesSamani para estimativa da $\mathrm{ET}_{0}$ no estado do Espírito Santo, Brasil. Revista Brasileira de Engenharia Agrícola e Ambiental. v.23, n.3, p.153-159, 2019. https://doi.org/10.1590/18071929/agriambi.v23n3p153-159

VESCOVE, H. V.; TURCO, J. E. P. Comparação de três métodos de estimativa da evapotranspiração de referência para a região de Araraquara-SP. Engenharia Agrícola, v. 25, n. 3, p. 713-721, 2005.

VISWANADHAM, Y.; SILVA FILHO, V.P.; ANDRÉ, R. G. B. The Priestley-Taylor parameter $\alpha$ for the Amazon forest. Forest Ecology and Management. v.38, p.211-225, 1991.

WILLMOT, C. J.; ACKLESON, S. G.; DAVIS, R. E.; FEDDEMA, J. J.; KLINK, K. M.; $\quad$ LEGATES, D. R.; O'DONNELL, J.; ROWE, C. M. Statistics for the evaluation and comparison of models. Journal of Geophysical Research, v. 90, p. 8995-9005, 1985. 